\title{
Implementation of OSPF on a Network Using GNS3 Tool
}

\author{
Apoorva N S ${ }^{1}$, Namratha $\mathbf{H}^{2}$, Rishik A Sunadoli ${ }^{3}$, Mariya Smitha $C^{4}$, Anil Umesh ${ }^{5}$ \\ Student, Department of TCE, DSCE, Bangalore, India ${ }^{1,2,3,4}$ \\ BE, Department of CSE, SJCE, Mysore, India ${ }^{5}$
}

\begin{abstract}
A Computer Network consists of many interconnected nodes, sharing resources linked through communication channels. Routers are placed at the nodes, forwarding the packets in the network. The packets are guided to their destination through the shortest path as defined by the dynamic routing protocol configured on the routers. In this paper, we have considered a network consisting of routers placed at the nodes, linked by serial interfaces. OSPF is configured on the network for the packets to reach the destination through the shortest path. Open Shortest Path First (OSPF) is a dynamic and classless routing protocol. We have used Variable Length Subnet Masking (VLSM), during IP addressing in the network. The network is password protected and encrypted. Any router can be remotely accessed using Telnet. We have used GNS 3 to build the situation and solve the problem. In this paper we also illustrate the setting of CTY, TTY, AUX and VTY password, create interface loopbacks to test the advertisement by OSPF routing protocol configured on each router and other aspects of OSPF routing protocol.
\end{abstract}

Keywords: Open Shortest Path First (OSPF) routing protocol, Autonomous System (AS), CTY, TTY, AUX and VTY, password protected, VLSM (Variable Length Subnet Masking), encrypted and GNS 3.

\section{INTRODUCTION}

A network can be classified into the following based on their span

- $\quad$ PAN- Personal Area Network

- $\quad$ LAN- Local Area Network

- WAN- Wide Area Network

- $\quad$ MAN- Metropolitan Area Network

- $\quad$ Global Network

Open Shortest Path First Routing Protocol (OSPF) configured on routers to effectively move packets around the computer network. OSPF uses the Dijkstra shortest path first algorithm to determine the shortest path in the network. OSPF follows the Link-state routing protocol.

- $\quad$ Each router establishes a relationship-an adjacency-with each of its neighbors.

- $\quad$ Each router sends Link State Advertisements (LSAs) and each OSPF area is flooded with Link State Advertisements

- $\quad$ Each router stores a copy of all the LSAs it has seen in a database.

- $\quad$ The completed topological database, called the Link-state database, describes a graph of the internetwork. Using the Dijkstra algorithm [1] the shortest path is calculated and routing table is updated.

The OSPF process builds and maintains three separate tables: [2]

- $\quad$ Neighbor table

- Topology table

- $\quad$ Routing table

\section{Problem statement}

To build a communication network consisting of routers at the nodes, forwarding packets to intended destination. To configure Open Shortest Path First (OSPF) routing protocol on the network routers. VLSM IP addressing scheme. [3] The network needs to be password protected and encrypted. Setting of CTY, TTY, AUX and VTY password, create interface loopbacks to test the advertisement by OSPF routing protocol configured on each router. Telnet protocol to remotely access the target router(s). 


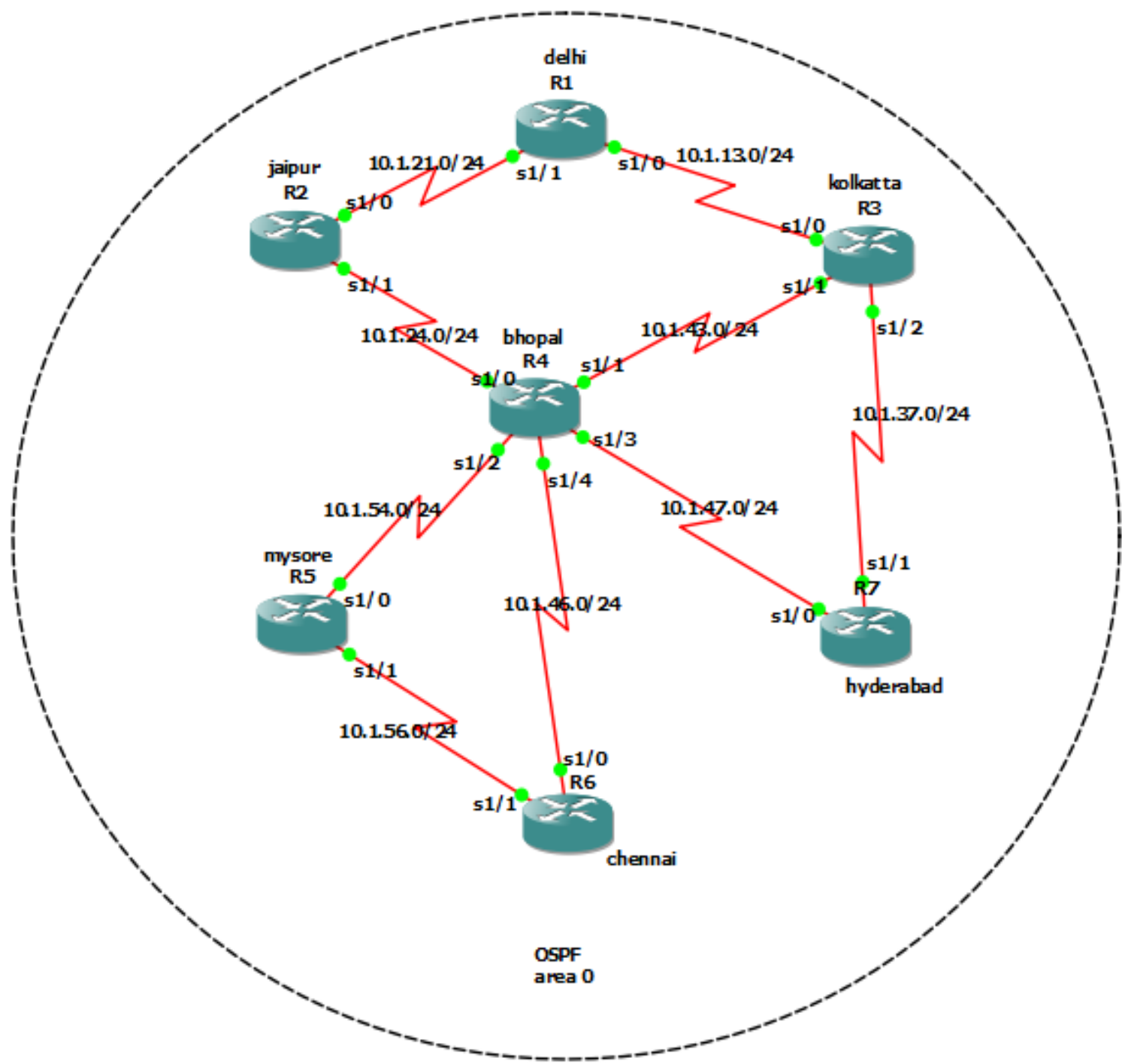

Figure 1 shows the network of interest.

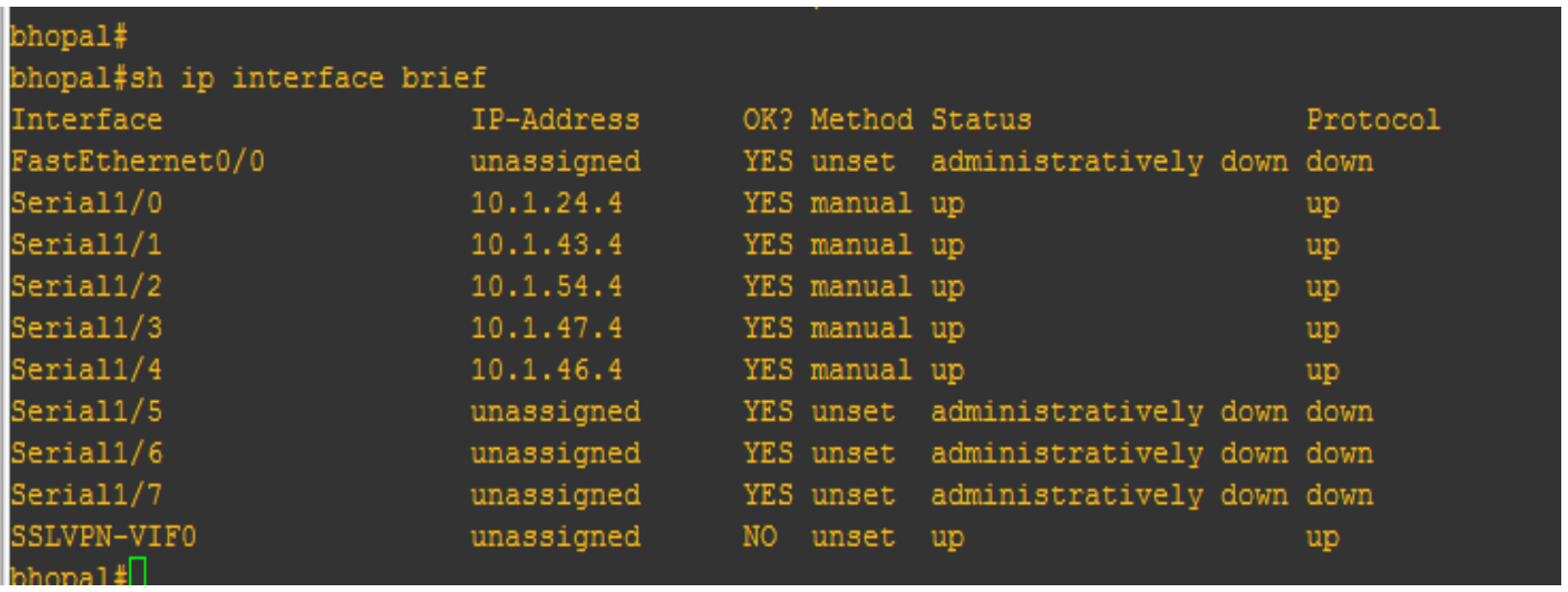

Figure 2 shows the router at bhopal and all its serial port interfaces. 
Vol. 8, Issue 5, May 2019

\section{NETWORK}

We have considered a network as shown in figure 1. OSPF area 0 is configured within the AS. Now each router communicate with each other by sending packets based on Link-state routing protocol and Dijkstra algorithm to find the shortest path from source to a specific destination. In the above network, the routers R1, R2, R3, R5, R6 and R7 is connected in star toplogy to router $\mathrm{R} 4$ which is the central router and the most powerful router.A central router is selected in such a way that it is physically equidistant from all other routers. Further R3 is connected to R1 and R7, R6 to R5 and R1 to R2. These connections may be wired or wireless, CTY or TTY or AUX or VTY lines or underground. Figure 2 shows the router at bhopal and all its serial port interfaces. Figure 3 shows the router at kolkata and all its serial port interfaces. 3.3.3.3/24 and 33.33.33.33/24 are the interface loopback 0 and interface loopback 1 of router at kolkata respectively.

\section{A. $\quad$ Setting CTY,TTY,AUX and VTY passwords}

\section{- CTY line}

The CTY line-type is the console port. On any router, it appears in the router configuration as line con 0 and in the output of the show line as cty. It is mainly used for local system access using a console terminal.

\section{- $\quad$ TTY lines}

TTY lines are asynchronous lines used for inbound or outbound modem and terminal connections and can be seen in a router or access server configuration as line $\mathrm{x}$. The specific line numbers are a function of the hardware built into or installed on the router or access server.

\section{- $\quad$ VTY lines -}

VTY line called the Virtual Terminal Lines of the router, used solely to control inbound telnet connections. There is no hardware associted with them.

- $\quad$ AUX line - It is the auxilary port.

Figure 4 shows line con 0 , line aux 0 and line vty 04 and password protection on CTY and VTY lines.

B. Telnet and ping

Figure 5 shows the effort to ping to IP address 10.1.56.5 from chennai and was successful. It also shows an effort by router at chennai to telnet IP address 10.1.47.4 to get remote acccess to router at Bhopal through VTY lines protected using password. After telnet an access to configure the router at bhopal is obtained. Ping is done to check the connectivity in a network by sending 5 packets. If the ping is successgul, meaning all 5 packets were successfully echoed. There is proper network connection else there will be connection error and needs to be taken care of.

\section{C. $\quad$ OSPF routing protocol [4]}

Figure 6 shows the router at Bhopal configured with OSPF routing protocol using show run command. Similarly all other routers are configured in the same manner. Interface loopbacks are created to test the advertisement by OSPF routing protocol configured on each router. Figure 7 shows an effort by router at bhopal to ping to interface loopback 0 (3.3.3.3/24) at R3 (kolkata) to check proper OSPF routing and route advertisement. We were successful in our attempt. All 5 packets successfully sent and received with a average round trip time of $374 \mathrm{~ms}$ and with a 2 second timeout.

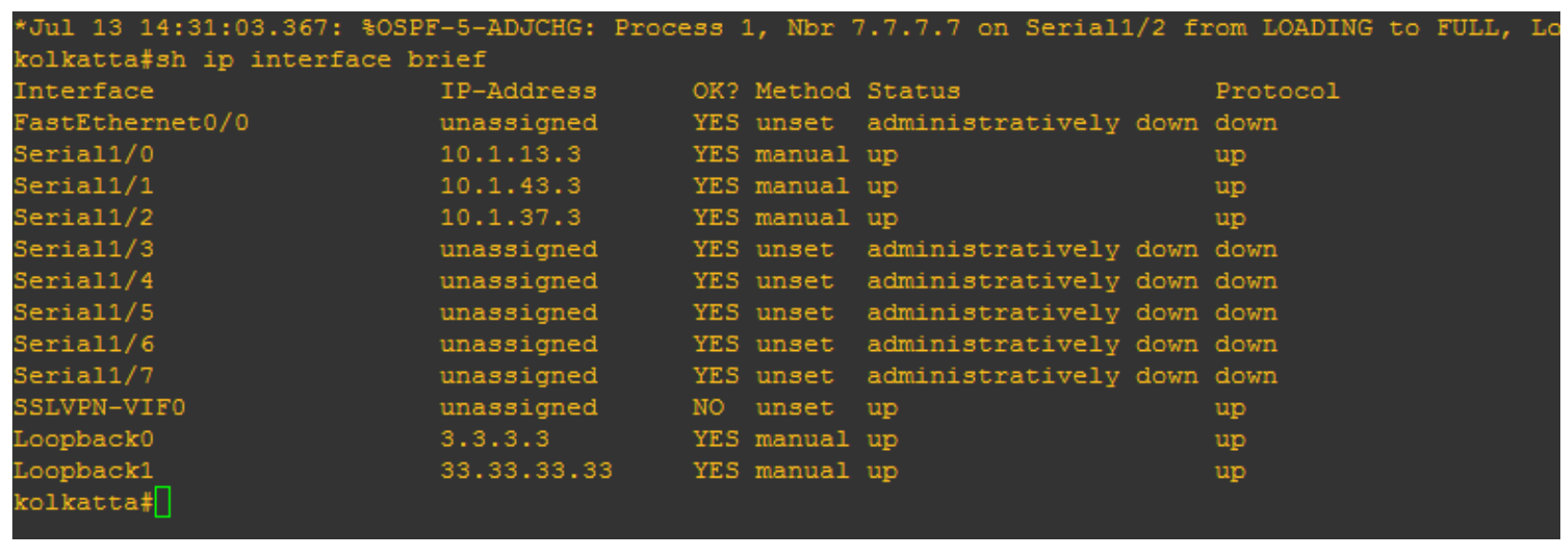

Figure 3 shows the router at kolkata and all its serial port interfaces. 


\section{International Journal of Advanced Research in Computer and Communication Engineering}

Vol. 8, Issue 5, May 2019

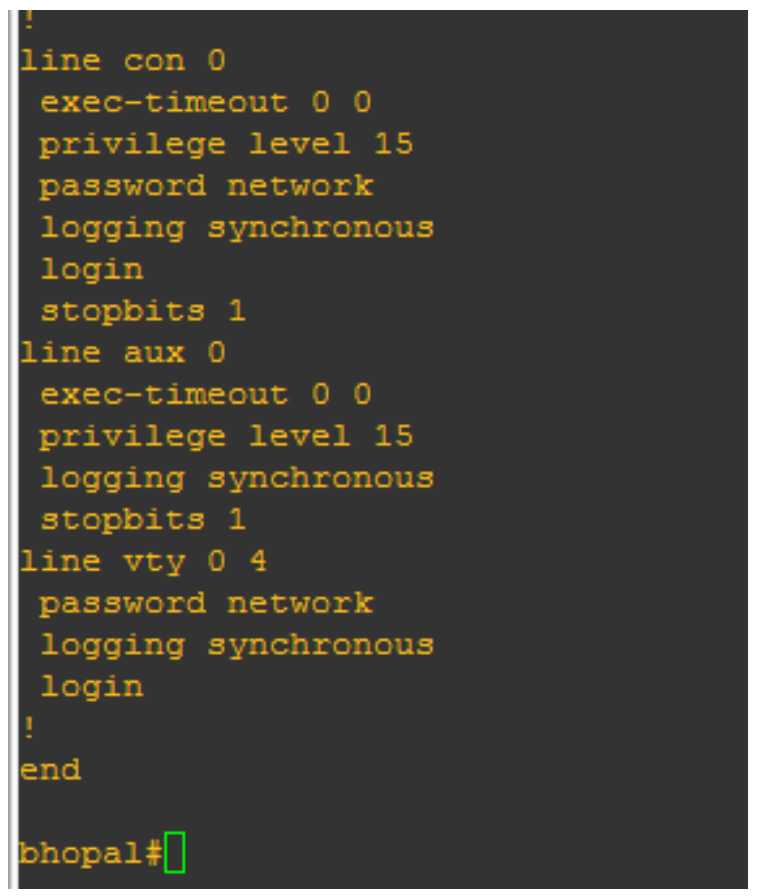

Figure 4 shows line con 0 , line aux 0 and line vty 04 and password protection on CTY and VTY lines.
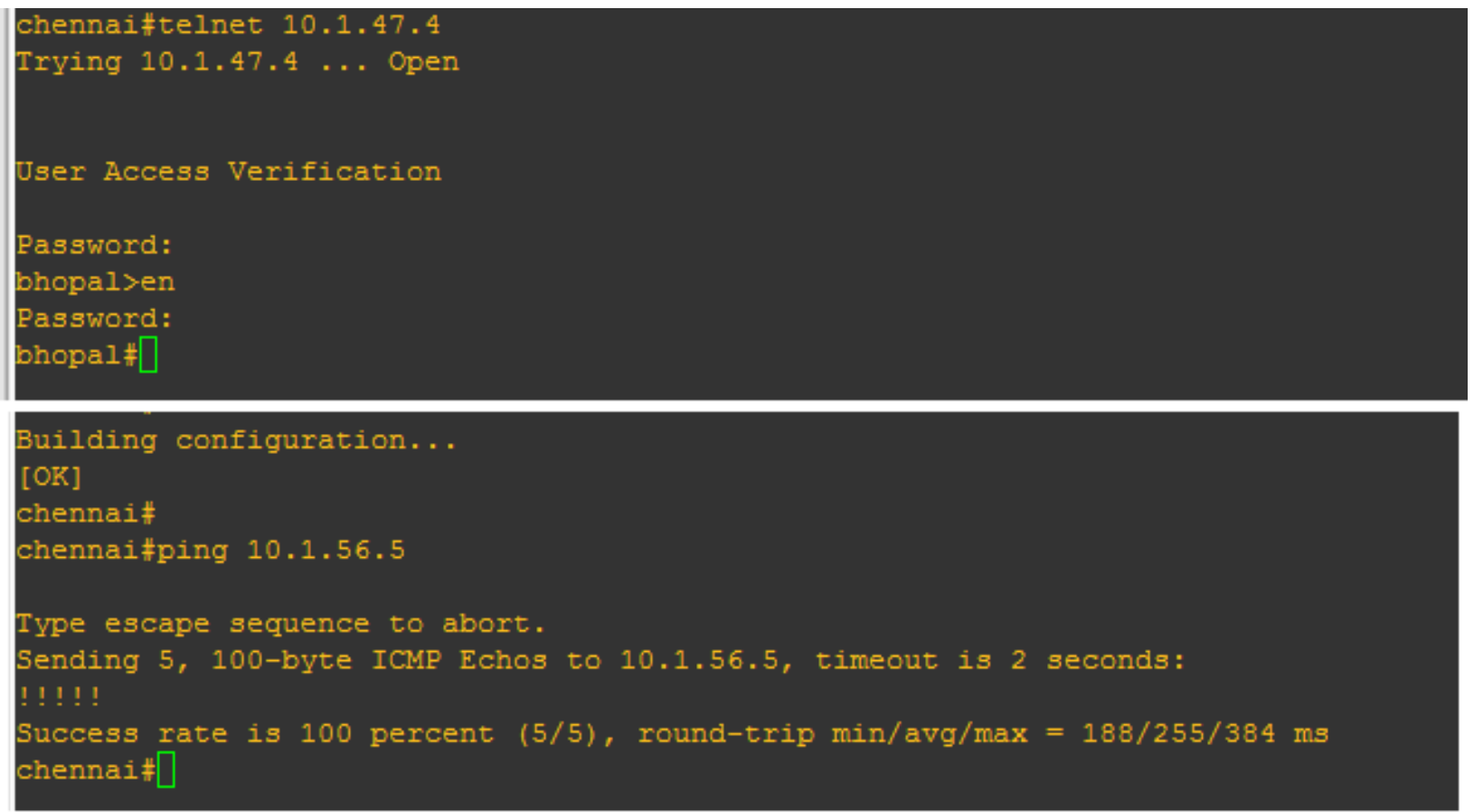

Figure 5 shows the effort to ping to IP address 10.1.56.5 from chennai and was successful.

\section{CONCLUSIONS}

In this paper efforts were made to build a communication network, consisting of routers at different nodes and configured with OSPF to effectively forward / route the packet along the network to intended destination. Various tests were conducted on the network to check the active participation of each router and the links interconnecting them. ICMP packets successfully echoed across the network indicating 100\% success rate during PING. VTY, CTY and line console passwords were successfully set and the packets encrypted. On Telnet we were able to take remote access through VTY lines. 


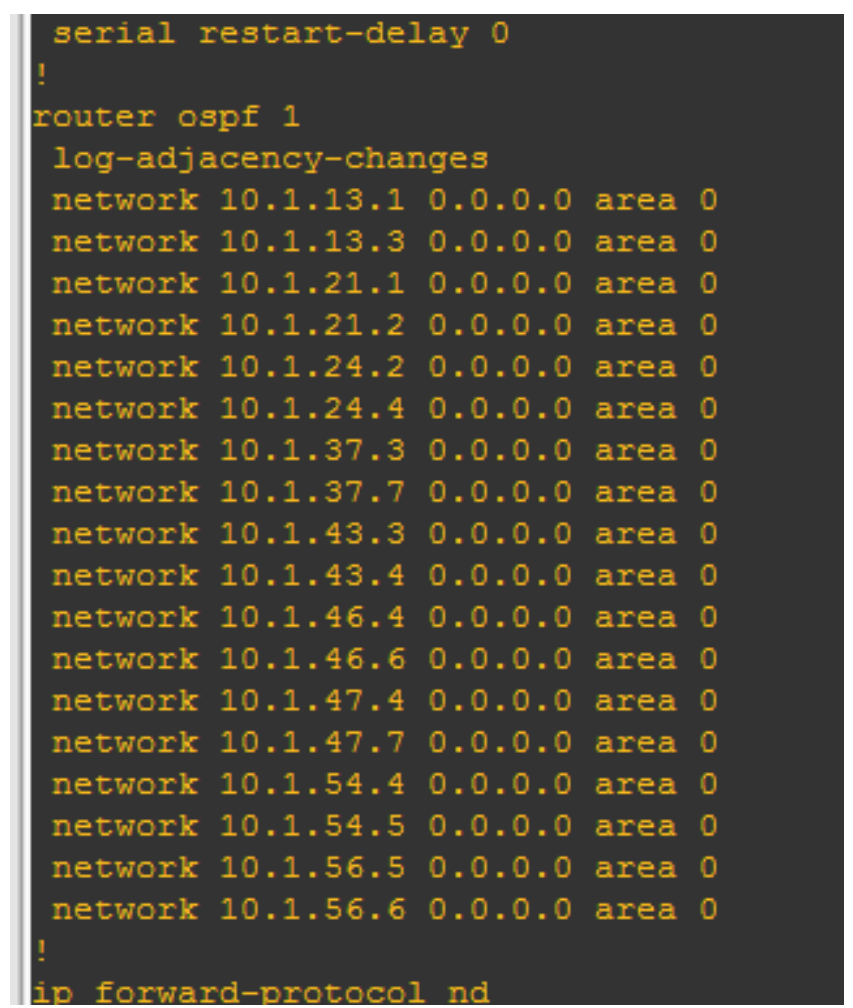

Figure 6 shows the router at Bhopal configured with OSPF routing protocol using show run command.

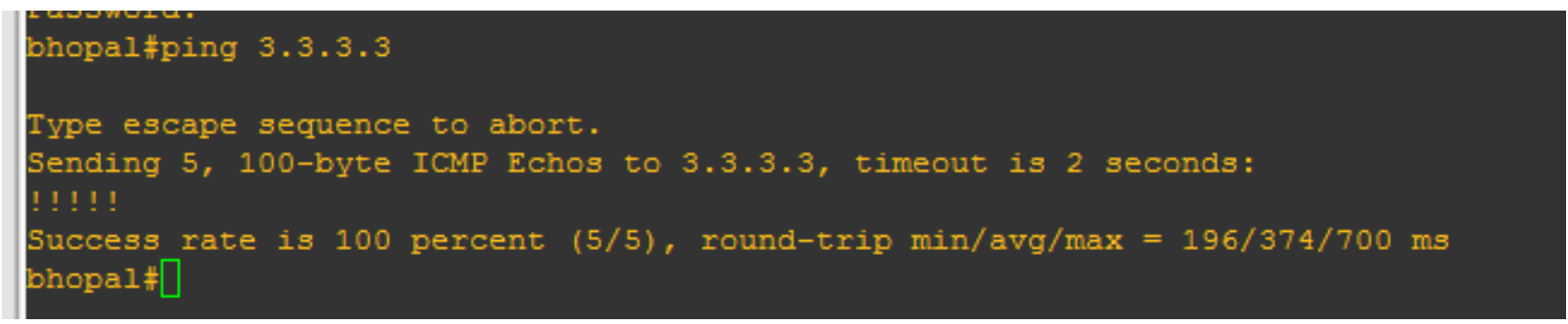

Figure 7 shows an effort by router at bhopal to ping to interface loopback 0 (3.3.3.3/24) at R3 (kolkata) to check proper OSPF routing and route advertisement.

\section{REFERENCES}

[1]. [1] DIJKSTRA'S ALGORITHM- math.mit.edu/ rothvoss/18.304.3PM/Presentations/1-Melissa.pdf

[2] OSPF Design Guide - Cisco- https://www.cisco.com/c/en/us/support/docs/ip/open-shortest...ospf/7039-1.html

[3]. [3] IP Addressing Guide - Cisco

[4]. https://www.cisco.com/c/dam/en/us/solutions/collateral/.../sba_ipAddr_dg.pdf

[5]. [4] Realization of Virtual Link on a Network- IJARCCE, DOI 10.17148/IJARCCE.2018.7912

\section{BIOGRAPHY}

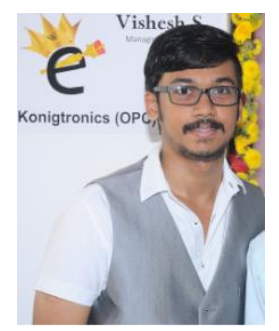

VISHESH S born on $13^{\text {th }}$ June 1992, hails from Bangalore (Karnataka) and has completed B.E in Telecommunication Engineering from VTU, Belgaum, Karnataka in 2015. He also worked as an intern under Dr. Shivananju BN, former Research Scholar, Department of Instrumentation, IISc, Bangalore. His research interests include Embedded Systems, Wireless Communication, BAN and Medical Electronics. He is also the Founder and Managing Director of the corporate company Konigtronics Private Limited. He has guided over a thousand students/interns/professionals in their research work and projects. He is also the co-author of many International Research Papers. He is currently pursuing his MBA in e-Business and PG Diploma in International Business. Presently Konigtronics Private Limited has extended its services in the field of Software Engineering and Webpage Designing. Konigtronics also conducts technical and non-technical workshops on various topics. 